\title{
International Journal of Aquatic Research and Education
}

$2-1-2010$

\section{Aquatic Fitness Research Poster and Presentation Abstracts}

Aquatic Exercise Association International Aquatic Fitness Conference

Follow this and additional works at: https://scholarworks.bgsu.edu/ijare

How does access to this work benefit you? Let us know!

\section{Recommended Citation}

Aquatic Exercise Association International Aquatic Fitness Conference (2010) "Aquatic Fitness Research Poster and Presentation Abstracts," International Journal of Aquatic Research and Education: Vol. 4: No.

1, Article 11.

DOI: https://doi.org/10.25035/ijare.04.01.11

Available at: https://scholarworks.bgsu.edu/ijare/vol4/iss1/11

This Abstract is brought to you for free and open access by the Journals at ScholarWorks@BGSU. It has been accepted for inclusion in International Journal of Aquatic Research and Education by an authorized editor of ScholarWorks@BGSU. 


\title{
Aquatic Fitness Research Poster and Presentation Abstracts
}

\author{
Aquatic Exercise Association International Aquatic Fitness \\ Conference \\ Orlando, FL: May 12-16, 2009
}

\section{Electromyographic Responses Induced by Superficial Electrodes Isolation and by Immersion}

\author{
Cristine Lima Alberton, Eduardo Marczwski Silva, Eduardo Lusa Cadore, Marcelo \\ Coertjens, Paulo Otto Beyer, Luiz Fernando Marocco, Luiz Fernando Martins Kruel, \\ Federal University of Rio Grande do Sul, Porto Alegre, Brazil
}

The purpose of the present study was to verify the electromyographic (EMG) signal alterations during isometric actions performed in water and on land. First, 8 young women $(23 \pm 1.0 \mathrm{yrs})$ performed maximal voluntary isometric actions in a isokinetic dynamometer to record the peak torque for knee extensors and the EMG signal of the vastus lateralis muscle $\left(90^{\circ}\right.$ flexion knee) with and without superficial electrodes isolation. Subsequently, another maximal isometric action was performed against fixed resistance on land and in water immersion. For statistical analysis a paired $t$ Test was used, with $\mathrm{p}<0.05$ (SPSS V11.0). No significant difference was found between the vastus lateralis root mean square (RMS) EMG values for with $(0.560 \pm 0.118 \mathrm{mV})$ and without $(0.538 \pm 0.110 \mathrm{mV})$ isolation situations $(\mathrm{p}$ $=0.306)$, for similar peak torque values $(\mathrm{p}=0.191)$. The same results were found comparing on land $(0.428 \pm 0.054 \mathrm{mV})$ and in water immersion $(0.388 \pm 0.105 \mathrm{mV})$ situations $(p=0.446)$. The water-resistant adhesive taping did not alter the EMG signal. Based on this finding, we found no differences on RMS EMG values during maximal isometric actions performed both in water and on land, suggesting that the environment did not influence the RMS EMG amplitude. Keywords: aquatic exercise, EMG, peak torque

\section{Correlation Between Rating of Perceived Exertion and Physiological Variables During the Execution of Stationary Running in Water at Different Cadences}

Cristine Lima Alberton, Amanda Haberland Antunes, Stephanie Santana Pinto, Marcus Peikriszwili Tartaruga, Eduardo Marczwski Silva, Eduardo Lusa Cadore, Luiz Fernando Martins Kruel 
The purpose of the present study was to correlate the rating of perceived exertion (RPE) with cardiorespiratory and neuromuscular variables during the execution of stationary running in water at different cadences. The sample consisted of 12 apparently healthy women (age: $22.33 \pm 0.57$ years). During the assessment session, the subjects performed a maximal voluntary contraction of the muscles of interest on land and, after, they executed the stationary running exercise in water at three different cadences, 60, 80 and $100 \mathrm{bpm}$, each one for $4 \mathrm{~min}$ (5 min intervals). Pearson Product Moment Correlation and multiple linear regression were used, with $\mathrm{p}<0.05$. The analyses demonstrate high and significant correlations between RPE and heart rate $(\mathrm{HR})(\mathrm{r}=0.65 ; \mathrm{p}<0.001)$, RPE and $\%$ HR maximal $(\mathrm{r}=0.65 ; \mathrm{p}<0.001), \mathrm{RPE}$ and oxygen uptake $\left(\mathrm{VO}_{2}\right)(\mathrm{r}=0.60$; $\mathrm{p}=0.001), \mathrm{RPE}$ and $\% \mathrm{VO}$ maximal $(\mathrm{r}=0.71 ; \mathrm{p}<0.001)$ and $\mathrm{RPE}$ and ventilation $(r=0.77 ; p<0.001)$. However, there was no correlation for the EMGs of the vastus lateralis, biceps femoris, rectus femoris and semitendinosus muscles and RPE. With regard to the regression, the model was significant $(\mathrm{p}<0.001)$ and presented $r^{2}=0.79$, while the variables that best explained the RPE were $\% \mathrm{VO}_{2}$ maximal and ventilation. Hence, these results suggest a high association between the perception of exertion and cardiorespiratory variables, which was not the case with the neuromuscular variables evaluated in this study. Therefore, the Borg Scale of RPE can be used when prescribing stationary running exercise in water for young women.

\section{Cardiorespiratory Responses to Stationary Running at Different Cadences in Water and on Land}

\section{Cristine Lima Alberton, Marcus Peikriswili Tartaruga, Stephanie Santana Pinto, Eduardo Lusa Cadore, Eduardo Marczwski da Silva, Luiz Fernando Martins Kruel, Federal University of Rio Grande do Sul, Porto Alegre, Brazil}

The aim of the current study was to analyze heart rate (HR), oxygen uptake $\left(\mathrm{VO}_{2}\right)$, and percentage of maximal $\mathrm{HR}\left(\% \mathrm{HR}_{\max }\right)$ and $\mathrm{VO}_{2}\left(\% \mathrm{VO}_{2 \max }\right)$ responses to stationary running exercises performed at different cadences on land (LA) and in water (WA). The sample comprised 12 apparently healthy women $(22.33 \pm 0.57$ years). The exercise was performed in WA and LA environments (interval of 2 hours) at sub-maximal cadences of 60,80 and $100 \mathrm{bpm}$ for a period of $4 \mathrm{~min}$ at each cadence (interval of $5 \mathrm{~min}$ ). The data were analyzed using factorial ANOVA for mixed models, Tukey's post hoc and the Pearson Product Moment Correlation test, with $\mathrm{p}<0.05$ (SPSS version 13.0). Significant differences were found between the environments for all variables, with higher values for LA. Significant differences were found between the cadences for all variables. The correlations between HR and $\mathrm{VO}_{2}$ (WA: $\mathrm{r}=0.857 ; \mathrm{p}<0.001$ and LA: $\mathrm{r}=0.556 ; \mathrm{p}<0.001$ ) and $\% \mathrm{HR}_{\text {mx }}$ and $\% \mathrm{VO}_{2 \max }$ (WA: $\mathrm{r}=0.860 ; \mathrm{p}<0.001$ and LA: $\mathrm{r}=0.798 ; \mathrm{p}<0.001$ ) exhibited high and significant correlation coefficient values, especially for WA, suggesting that variable increased linearly with increases in cadence. The results suggest that cardiorespiratory responses to stationary running exercise are lower in WA, though they can be maximized by increasing the execution cadence. Keywords: water exercise, stationary water running, cadences 


\title{
Deep Water Running: Developing Training Intensities From Land Based Exercise
}

\author{
D. Donley, D. Bonner, M. Kampert, L. Sherlock, G. Hornsby, West Virginia Univer- \\ sity, School of Medicine, Department of Human Performance and Applied Exercise \\ Science, Morgantown, WV
}

Individuals engaging in water exercises such as deep water running (DWR), should determine their exercise intensities from fitness assessments performed with the same mode of exercise (i.e., DWR). However, land based exercise protocols are typically preferred by individuals who are moderate to high risk for disease. The purpose of this study was to compare measures of exercise intensity between DWR and treadmill (TM) exercise. Maximal TM and DWR exercise tests were performed on thirty healthy subjects ( 15 men, 15 women). The max TM test was administered a minimum of 48 hours prior to the max DWR test. Heart rate (HR), oxygen consumption $\left(\mathrm{VO}_{2}\right)$, and respiratory exchange ratio (RER) were measured. Resting $\mathrm{VO}_{2}\left(4.3 \pm 0.7 \mathrm{ml} \cdot \mathrm{kg}^{-1} \cdot \mathrm{min}^{-1}\right.$ for TM vs $6.0 \pm 1.3 \mathrm{ml} \cdot \mathrm{kg}^{-1} \cdot \mathrm{min}^{-1}$ for DWR) and resting RER $(0.88 \pm 0.08$ for TM vs $0.99 \pm 0.09$ for DWR) were significantly lower $(\mathrm{p}<0.001)$ during the TM test compared to the DWR test. $\mathrm{HR}_{\max }(190 \pm$ $11 \mathrm{bpm}$ for TM vs $174 \pm 10 \mathrm{bpm}$ for DWR $), \mathrm{VO}_{2 \max }\left(45.3 \pm 7.4 \mathrm{ml} \cdot \mathrm{kg}^{-1} \cdot \mathrm{min}^{-1}\right.$ for TM vs $37.3 \pm 7.3 \mathrm{ml} \cdot \mathrm{kg}^{-1} \cdot \mathrm{min}^{-1}$ for DWR) and Max RER $(1.24 \pm 0.08$ for TM vs $1.15 \pm 0.09$ for DWR) were significantly lower $(\mathrm{P}<0.001)$ for DWR compared to TM. $\mathrm{HR}_{\max }$ during the TM test had a significant positive correlation with $\mathrm{HR}_{\text {max }}$ during DWR $(r=0.77, \mathrm{p}<0.01)$ and accounted for $59 \%$ of the variance for $\mathrm{HR}_{\max }^{\text {max }}$ during DWR. We concluded that $\mathrm{HR}_{\max }$ during DWR was decreased by $16 \pm 7 \mathrm{bpm}$ compared to max TM testing. $\mathrm{VO}_{2 \max }$ was reduced by $17 \%$ during DWR compared to TM testing. Keywords: Water exercise, deep water running, exercise intensities

\section{Effects of the Aquatic and Land Plyometric Methods on Vertical Jump and the Delayed Onset Muscle Soreness Perception in Soccer Players}

Renato Tavares Fonseca ${ }^{1}$; Antonio Aborrage1; Sérgio Gregório da Silva²; Paula Roquetti Fernandes ${ }^{4}$; José Fernandes Filho3; Estélio H.M.Dantas ${ }^{1}$

${ }^{1}$ Castelo Branco University - Brazil; ${ }^{2}$-Federal University of Paraná - Brazil; ${ }^{3}$-Federal University of Rio de Janeiro (UFRJ)-Brazil; ${ }^{4}$-Center of Excellence in Physical Evaluation - Brazil

The use of resources that increase muscular power in sports have turned to plyometric exercise as one of the main methods for the development of the vertical jump. This is in spite of the fact that it presents potential risk for the appearance of lesions, mainly during eccentric activities. Researchers have postulated that plyometric exercises accomplished in the aquatic environment can reduce the potential risk of muscular soreness and of lesions on the human muscle skeletal system. The objective of this study was to compare the aquatic 
and land plyometric methods on the vertical jump and the delayed onset of muscle soreness perception (DOMS) in soccer players. Subjects who participated in the study included 23 soccer players from the Fluminense Futebol Clube, in the age group between 16 and 18 years old. An experimental group of jumps in the water $($ APT $)(n=09 ; 16.4 \pm 0.4$ years; $68.3 \pm 7.54 \mathrm{~kg} ; 179.75 \pm 8.13 \mathrm{~cm})$, an experimental group of land jumps (LPT) $(\mathrm{n}=08 ; 16.5 \pm 0.5$ years; $68.2 \pm$ $7.8 \mathrm{~kg} ; 177 \pm 7.4 \mathrm{~cm})$ and a control group $(\mathrm{CG})(\mathrm{n}=06 ; 16.7 \pm 0.6 y$ ears; 61.2 $\pm 6.5 \mathrm{Kg} ; 171.43 \pm 5.75 \mathrm{~cm}$ ), participated in the research. The experimental groups were submitted to an identical training program, for six weeks, twice a week, totalling 944 jumps. The vertical jump was evaluated on a leap jump platform, and the response of DOMS perception was obtained by the Ratio-10 Borg Scale. In the intra-groups comparison, there was significant increase for the vertical jump in the LPT group $(\mathrm{p}=0.001)$, as well as in the APT group ( $\mathrm{p}$ $=0.000)$. There was a significant reduction $(\mathrm{p}<0.05)$ in the foot contact with the floor of the APT group, when compared to LPT and CG groups. There was a significant reduction in DOMS for the APT group compared to the LPT group $(\mathrm{p}=0.001)$. It can be concluded that plyometric aquatic training can increase vertical jump and significantly reduce the perception of DOMS in soccer players. Keywords: plyometric training, muscular pain perception, vertical jump and jumping training in soccer.

\title{
American Red Cross Learn to Swim Program: Outcomes Among African American Children in Charleston, SC
}

\author{
Nancy Haynsworth, M.A., and Dr. Phillip Bogle, Ph.D., Department of Health, \\ Exercise and Sport Science, The Citadel, Charleston, SC
}

Drowning ranks second as the leading cause of death for children up to 14 years of age. Drowning rates are substantially higher for African-Americans. In general, African-American children are much more likely to drown than Caucasian children. Also, among all racial groups, African-Americans report the most limited swimming ability. This study addressed the existing disadvantage in drowning rates among African-Americans by sponsoring a six-week American Red Cross (ARC) Swimming Program. The study was designed to increase swimming skills among African-American children. Children from five to ten years of age were provided approximately six hours of swimming instruction. Pre-post tests were given to all students as they entered and exited the study. McNemar's test was used to determine whether a treatment effect existed using the ARC Program. The number of all children who initially failed and then passed $(n=41 ; 85.4 \%)$ the Level I pre-post test swim program was significantly different from the number who could not ( $\mathrm{n}$ $=2 ; \mathrm{p}<.001$ ). Although African-Americans have a higher incidence of drowning than Caucasians, the African-American children who participated in this study were able to master the same skills as other ethnic groups. Future studies should examine the impact of skill enhancement and the decreased drowning rates of the African-American population. 


\title{
Incidence of Lesions Among Apprentices of Indoor Cycle and Aquatic Cycle
}

\author{
Antonio Fernando F. de Sá Junior; Roxana Macedo Brasil; Ana Cristina Lopes \\ Barreto, Rio de Janeiro
}

The aim of this study was to compare the incidence of lesions among apprentices of indoor and aquatic cycling exercises. The descriptive and qualitative research was performed with a group of volunteers made up by 207 participants of both genders; 149 of those were indoor cycling apprentices (IC: $30.10 \pm 8.21$ years old) and 58 were aquatic cycling apprentices (AC: $30.43 \pm 8.4$ years old) in sport clubs in the municipal district of Rio de Janeiro. A questionnaire with 12 questions was used, elaborated in the Likert scale. From the frequency analysis it was observed that the IC group attended classes four times a week while the AC group only three times. In the IC group, besides cyclism, eighty-eight subjects performed other modalities of exercise; eighteen subjects of the AC group did the same. Most of the volunteers never regulated the equipment, but in the IC group, the largest obtained frequency was that of the volunteers that regulated the bicycle before beginning the class. In both groups the largest observed frequency belonged to individuals that felt some discomfort or pain during the class. In the AC group most of the subjects felt severe level of pain while in the IC group the largest frequency was that of individuals with moderate pain level. The pain recurrence presented a fortnight frequency in the AC practicing group and daily frequency in the IC practicing group. In the AC group, low back pain was the most frequent type of lesion while in the IC group inflammations were. As for the lesion spot, the AC practicing group presented a larger pain frequency in the shoulders and the IC practicing group presented pain in the spine. In both groups the subjects sought treatment in an attempt to recover from the lesions. Based on the results, it was possible to identify that for the volunteer group in both modalities the lesion incidence was low although the feeling of discomfort and pain was high. Keywords: aquatic exercise, indoor cycling, aquatic cycling, risk of injury

\section{Cardiorespiratory Responses of Young Women During a Hydrogymnastics Exercise Performed With and Without Horizontal Movement on Land and in Aquatic Environment in a Deep and in a Shallow Swimming Pool}

\author{
Ana Carolina Kanitz; Eduardo Marczwski da Silva; Cristine Lima Alberton; Luiz \\ Fernando Martins Kruel, Laboratory of Research on Exercise, Federal University \\ of Rio Grande do Sul, Porto Alegre, Brazil
}

The aim of the current study was to compare cardiorespiratory responses during a hydrogymnastics exercise performed with and without displacement on land (LE) and in the aquatic environment in a deep (DS) and in a shallow swimming 
pool (SS). Six young women performed the exercise for four minutes in each environment (LE, DS and SS) and situation (with and without displacement) at a cadence of $80 \mathrm{bpm}$. The exercise consisted of hip flexion/extension with the arms simulating a running movement. Heart rate (HR) and oxygen uptake (VO2) were verified during the last minute for each exercise and rate of perceived exertion (RPE) was collected at the end of the exercise. Energy expenditure (EE) was calculated from the product between the absolute vo 2 value and the equivalent caloric expenditure of $5 \mathrm{kcal} . \mathrm{min}-1$. Variable comparisons used ANOVA two-way for repeated measures with factors of the environment and situation $(\mathrm{p}<0.05)$. For all cardiorespiratory variables analyzed, SS exercise responses were lower than that found for LE. Although, no difference was observed in the exercise performed on LE or in DS, except for the HR, which was lower during DS. For the different situations, a higher HR value was found during exercise with displacement when compared with exercise without displacement in the DS. These findings suggest the possibility to perform the exercise analyzed with similar energy expenditure and lower HR in DS when compared with exercise in LE. It is valuable for people that need similar energy expenditure yet lower cardiovascular overload during aquatic exercise. Keywords: water exercise, hydrogymnastics, energy expenditure, heart rate

\title{
Comparison of the Effect of the Same Cycling Classes Protocol in the Responses of Heart Rate in Relation to Environment and Gender
}

\author{
Joao Manuel Lages, Roxana Macedo Brasil, Grace Barros, Andrea Ferreira, \\ Madalena Souza, Ana Christina Lopes Barreto, Rio de Janeiro
}

Among the different strategies for measuring intensity control, heart rate (HR) has been used as one of the main physiologic variables related to exercise prescription. The purpose of this study was to compare the effect of the same protocol on the responses of $\mathrm{HR}$ in relation to environment and gender. Ten participants, 5 men $(24.20 \pm 5.21$ years; $175.40 \pm 6.84 \mathrm{~cm} ; 73.30 \pm 11.34 \mathrm{~kg} ; 67.00 \pm 9.00 \mathrm{bpm}$ in rest) and 5 women $(26.40 \pm 5.89$ years; $163.60 \pm 4.72 \mathrm{~cm} ; 58.30 \pm 5.97$ $\mathrm{kg} ; 71.00 \pm 8.00 \mathrm{bpm}$ in rest) engaged in similar exercise testing regiments. The protocol lasted 35 minutes, split in 5 stages in relation to body position and cadence of the pedals. An independent T-test was used to determine the differences between men and women. The study used a level of significance of $\alpha \leq 0.05$. The average HR between indoor cycling (IC) and aquatic cycling (AC) showed significant differences between some of the five stages: 2 (73.93 $\pm 11.72,72.75$ $\pm 16.71 \mathrm{bpm}) ; 3(81.41 \pm 6.08 ; 82.96 \pm 9.10 \mathrm{bpm})$ and $5(80.82 \pm 8.14 ; 79.31 \pm$ $14.51 \mathrm{bpm})$, respectively. The comparison in relation to gender showed that the women presented significant differences in more stages than did the men under both conditions (IC and AC). The same protocol generated different results for the different environments and gender. Keywords: indoor cycling; aquatic cycling; heart rate; gender 


\section{The Effects of High Volume Aquatic Plyometric Training on Vertical Jump, Muscle Power, and Torque}

Adam Ploeg, ATC; Travis Dibbet, ATC; Michael G. Miller, EdD, ATC, CSCS; Jennifer O'Donoghue, PhD; HPER Department, Western Michigan University, Kalamazoo, MI; William Holcomb, PhD, ATC, CSCS*D, University of Nevada Las Vegas; David Berry, PhD, ATC, Weber State University

The purpose of this study was to examine the effects of high volume aquatic-based plyometrics versus lower volume land and aquatic plyometric training on vertical jump (VJ), muscular peak power and torque in the dominant knee. Thirty-nine participants were randomly assigned to 1 of 4 groups: aquatic group 1 (APT1), aquatic group 2 (APT2), land group (LPT1), and control group (CON). All groups performed a 6-week plyometric training program. The APT1 and LPT performed the same volume of training where, APT2 doubled the volume. All participants were pre- and post-tested on performance variables. A 4 (group) $\times 2$ (time) ANOVA with repeated measures was used to determine differences among the performance variables. We found no significant differences between groups for all tested variables. APT2 showed greater descriptive average increase among the performance variables. The high volume aquatic plyometric protocol appears to be useful to help increase performance and minimize muscle soreness. Key Words: performance variables, water exercise, jump training, plyometrics

\section{Cardiorespiratory and Body Composition Responses to a Water Exercise Program for Athletes}

\section{J. Whitehill Jr., N.L. Constantino, M.E. Sanders, School of Community Health Sciences, University of Nevada, Reno}

The purpose of this study was to determine the effectiveness of a water-based exercise program on improving cardiovascular fitness and body composition of land-based competitive athletes during off-season training. Twenty-nine healthy, competitive athletes (aged $23.7 \pm 6.5$ years) were recruited to either a water exercise (WE, $n=14)$, or control $(C, n=15)$ group. The WE group trained a minimum of 3 times per week, for 90 minutes each session, performing aquatic aerobic activities, resistance exercises, and stretching. WE activities included shallow, deep and underwater running, jumping, cadence work, interval sets, and shallow-water cycling. The control group maintained land-based, off-season training and recorded all fitness activity. The duration of the program was 9 weeks. Body composition and cardiovascular measurements were obtained at the beginning and at the end of the training period. After 9 weeks of training, a two-sample $t$ test revealed that all measures improved the body significantly $(\mathrm{p}<$ $0.05)$ for the WE group compared to the $\mathrm{C}$ group. Body weight decreased (1.0 \pm $2.6 \mathrm{~kg}$, vs $-0.7+5.9 \mathrm{~kg})$; percent body fat decreased $(1.22 \pm 0.9$, vs $-0.37 \pm 1.8)$ 
100

Abstracts

and lean body mass increased $(0.12 \pm 3.5 \mathrm{~kg}$ vs $-0.71 \pm 3.4 \mathrm{~kg})$, respectively. Maximal responses for $\mathrm{V}_{2 \max }(5.7 \pm 1.5 \mathrm{ml} / \mathrm{kg}$ min vs $0.9 \pm 4.1 \mathrm{ml} / \mathrm{kg} \mathrm{min})$ were significantly higher ( $\mathrm{p} \leq 0.001)$, for the WE group compared to the control group, respectively. No injuries were reported during the program. This water exercise program conducted in the off-season resulted in improvements in body composition and cardiovascular fitness for a variety of competitive land-based athletes. Water exercise appears to be a promising training option for healthy competitive athletes to safely and significantly improve fitness. Keywords: Water exercise, cardiovascular fitness, body composition 